\title{
Almost periodicity in time-dependent and state-dependent delay differential equations
}

\author{
El Hadi Ait Dads ${ }^{1}$, Brahim Es-sebbar ${ }^{2}$, and Lahcen Lhachimi ${ }^{3}$ \\ ${ }^{1}$ Université Cadi Ayyad, Faculté des Sciences Semlalia B.P. 2390 \\ ${ }^{2}$ Cadi Ayyad University Faculty of Science and Technology Gueliz \\ ${ }^{3}$ Université Cadi Ayyad Faculté des Sciences Semlalia
}

August 3, 2020

\begin{abstract}
We study the response of various linear and nonlinear differential equations to different kinds of forced oscillations, specially the periodic and almost periodic oscillations. A special attention is given to differential equations with time-almost periodic type and state-dependent delays. To the best of our knowledge, there are no results in literature that address this problem.
\end{abstract}

\section{Hosted file}

PAP_delay.pdf available at https://authorea.com/users/317558/articles/473737-almostperiodicity-in-time-dependent-and-state-dependent-delay-differential-equations 